\title{
Impact of health-related quality of life on survival after dialysis initiation: a prospective cohort study in Korea
}

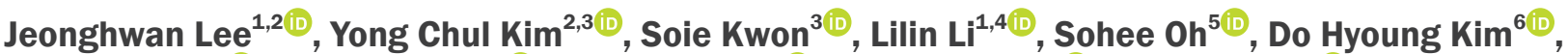

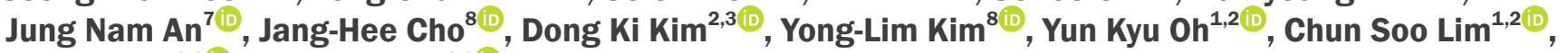 \\ Yon Su Kim ${ }^{2,3}$, Jung Pyo Lee ${ }^{1,2}$ iD \\ 'Department of Internal Medicine, Seoul Metropolitan Government Seoul National University Boramae Medical Center, Seoul, \\ Republic of Korea \\ ${ }^{2}$ Department of Internal Medicine, Seoul National University College of Medicine, Seoul, Republic of Korea \\ ${ }^{3}$ Department of Internal Medicine, Seoul National University Hospital, Seoul, Republic of Korea \\ ${ }^{4}$ Department of Intensive Care Unit, Yanbian University Hospital, Yanji, Jilin, China \\ ${ }^{5}$ Medical Research Collaborating Center, Seoul Metropolitan Government Seoul National University Boramae Medical Center, Seoul, \\ Republic of Korea \\ ${ }^{6}$ Department of Internal Medicine, Hallym University Kangnam Sacred Heart Hospital, Seoul, Republic of Korea \\ ${ }^{7}$ Department of Internal Medicine, Hallym University Sacred Heart Hospital, Anyang, Republic of Korea \\ ${ }^{8}$ Department of Internal Medicine, Kyungpook National University Hospital, School of Medicine, Kyungpook National University, \\ Daegu, Republic of Korea
}

Background: The effect of each health-related quality of life (HRQOL) component on hemodialysis prognosis has not been well studied. We aimed to investigate the clinical factors associated with HRQOL and the effect of HRQOL after dialysis initiation on long-term survival in an Asian population.

Methods: A total of 568 hemodialysis patients were included from a nationwide prospective cohort study. HRQOL was evaluated using the Kidney Disease Quality of Life (KDQOL) Short Form ${ }^{\mathrm{TM}} 1.3$ at 3 months after dialysis initiation. The effect of each KDQOL item score on mortality was analyzed. Multivariable Cox analysis was performed after adjusting for age, sex, modified Charlson comorbidity index, and causes of primary kidney disease.

Results: Old age, diabetes mellitus, high comorbidities, and low serum albumin levels were associated with poor physical health status. Decreased urine output was associated with both poor physical and mental health status. The scores of 3 indices in the kidney disease domain (effect of kidney disease, social support, and dialysis staff encouragement) showed significant associations with mortality, as did the 3 indices (physical function, physical role limitation, and body pain) in the physical health domain. Neither the 4 indices in the mental health domain nor the mental composite score showed a significant association with mortality. However, a high physical composite score was associated with decreased overall patient mortality $(P=0.003)$. The effect of physical composite score on survival was prominent among young or middle-aged groups.

Received April 30, 2020; Revised August 8, 2020; Accepted August 25, 2020

Editor: Young-Ki Lee, Hallym University, Seoul, Republic of Korea

Correspondence: Jung Pyo Lee

Department of Internal Medicine, Seoul National University Boramae Medical Center, Seoul National University College of Medicine, 20 Boramae-ro 5-gil, Dongjak-gu, Seoul 07061, Republic of Korea. E-mail: nephrolee@gmail.com

Copyright (C) 2020 by The Korean Society of Nephrology

(a) This is an open-access article distributed under the terms of the Creative Commons Attribution Non-Commercial License (http://creativecommons.org/ licenses/by-nc-nd/4.0/), which permits unrestricted non-commercial use, distribution, and reproduction in any medium, provided the original work is properly cited. 
Conclusion: Poor physical health status 3 months after hemodialysis start correlates significantly with overall mortality.

Keywords: Dialysis, Mental health, Mortality, Physical health, Quality of life

\section{Introduction}

Health-related quality of life (HRQOL) is defined as an individual's perceived physical and mental health status over time and includes conceptual domains related to physical, mental, and disease-related functional status. Correct assessment and improvement of patients' selfreported physical and mental health status are important factors for adequate disease management and high-level patient care. Poor HRQOL is often reported in patients with chronic diseases [1]. Dialysis patients have poor HRQOL compared with the general population [2]. The subjective physical and mental state of well-being, expressed as HRQOL, is a useful clinical index that predicts outcomes of hospitalization and mortality among patients with chronic kidney disease (CKD) and end-stage kidney disease (ESKD) requiring renal replacement therapy [3-6]. Increasing HRQOL with improving patients' subjective satisfaction and overall prognosis in dialysis patients has become an important goal of renal replacement therapy [7].

Two main domains, physical and mental health scores, are included in HRQOL and reveal the correlation of various clinical outcomes with overall survival [8-10]. There is a high prevalence of depression, of up to $30 \%$, among dialysis patients, and poor mental health status has been reported as a risk factor for hospitalization and mortality $[11,12]$. Appropriate treatment has been shown to improve the mental health level of dialysis patients [13]. Therefore, it is important to identify and assess the mental health status among dialysis patients. Low HRQOL status may also be due to the patients' poor physical health status [14]. In general, dialysis patients show low physical activity and physical function [15]. Impaired daily physical activity and physical performance are associated with anxiety and depression, and dialysis patients with low physical function were reported to have poor survival compared with those with better physical status $[16,17]$.

A difference in HRQOL status between ethnic groups among hemodialysis patients has also been reported [18].
Better HRQOL was reported in African American patients on hemodialysis than in their non-African American counterparts [19]. To date, the causes of racial disparities in HRQOL have not been well investigated. Racial disparities in HRQOL can result in differential effects on clinical outcomes [20,21]. The effects of HRQOL on the prognosis of dialysis patients have been reported in Caucasian and African American populations. Asian dialysis patients account for a significant proportion of dialysis patients worldwide and generally show better prognosis than other ethnic groups [22]. However, relatively few reports have investigated the association between HRQOL and survival among Asian dialysis patients [9]. Therefore, it is important to research the effects of HRQOL on the clinical outcomes and patient prognosis among Asian dialysis patients.

The Kidney Disease Quality of Life Short Form ${ }^{\mathrm{TM}} 1.3$ (KDQOL-SF ${ }^{\mathrm{TM}}$ 1.3; Supplementary Material 1, available online) is a self-administered questionnaire that can be used to assess HRQOL in patients on maintenance dialysis [23-25]. In this study, we utilized the KDQOL-SF ${ }^{\mathrm{TM}} 1.3$ to evaluate the HRQOL, including physical and mental health domains, and investigated the clinical factors associated with HRQOL and the association between each index in HRQOL and overall survival. We aimed to determine which components of the HRQOL are associated with patient prognosis and survival in dialysis patients and the effects of mental and physical health on mortality in an Asian hemodialysis population.

\section{Methods}

\section{Study participants}

The Clinical Research Center for End Stage Renal Disease (CRC for ESRD) cohort is a nationwide, multi-center, web-based, prospective cohort of CKD patients undergoing dialysis in South Korea [26,27]. The CRC for ESRD cohort started to register ESKD patients on dialysis in 31 hospitals in South Korea in July 2008. Patients who were 
at least 20 years old and began treatment with maintenance dialysis due to ESKD between July 2008 and May 2014 were eligible for this study. Patients in the database whose kidney function recovered after enrollment or patients whose clinical information and laboratory test results could not be gathered due to the discontinuation of follow-up were excluded. Among 1,850 adult patients who started maintenance dialysis, the participants who completed the baseline HRQOL questionnaire were enrolled in this study. Finally, 568 patients who received hemodialysis were included in the final analysis.

All patients participated voluntarily after providing written informed consent. The study was approved by the Institutional Review Board at each center and conducted in accordance with the Declaration of Helsinki 2013. All data were registered in the web-based database and extracted for outcome analysis. Baseline information at enrollment included age, sex, height, weight, body mass index, primary renal disease, type of dialysis, modified Charlson comorbidity index (mCCI), and laboratory data. The estimated glomerular filtration rate (eGFR) was calculated using CKD-Epidemiology Collaboration equations [28,29]. The mCCI was calculated for each patient at the initiation of dialysis [30,31]. Dialysis modality was defined as the modality 3 months after the first dialysis or the modality at dialysis initiation if death occurred before 3 months. Information about the HRQOL was gathered at 3 months after dialysis initiation and study enrollment to evaluate the prognostic impact of HRQOL on patients' survival.

\section{Patient and public involvement}

Patients were not involved in the design, recruitment, or any conduct of this research. All questionnaires and measured outcomes were described to the participants before enrollment and during the first interview by medical coordinators. All results from this cohort study will be provided to the participants via academic article, news, and mass media.

\section{Survey instruments}

The Korean version of KDQOL-SF ${ }^{\mathrm{TM}} 1.3$ was used to evaluate the HRQOL of ESKD patients 3 months after dialysis initiation [32]. The validity of HRQOL assessment using KDQOL-SF ${ }^{\mathrm{TM}} 1.3$ in patients with ESKD was con- firmed in previous studies [33,34].

The KDQOL-SF ${ }^{\mathrm{TM}} 1.3$ consists of 80 questions and all patients on dialysis were required to answer 79 of them (one question pertains to the type of dialysis: hemodialysis or peritoneal dialysis). The 79 questions include the follow: 35 items, generating 4 physical health indices (physical functioning, 10 items; physical role limitation, 4 items; pain, 2 items; and general health, 5 items) and 4 mental health indices (emotional well-being, 5 items; emotional role limitation, 3 items; social function, 2 items; and energy/fatigue, 4 items); 42 kidney diseasetargeted items, generating 10 indices (symptom/problem list, 12 items; effects of kidney disease, 8 items; burden of kidney disease, 4 items; work status, 2 items; cognitive function, 3 items; quality of social interaction, 3 items; sexual function, 2 items; sleep, 4 items; social support, 2 items; and dialysis staff encouragement, 2 items); 1 patient satisfaction item; and 1 overall health rating item.

Physical and mental composite scores were calculated using the 12 generic chronic disease items (the short form, SF-12) included in the 35 items generating 4 physical and 4 mental health indices. It takes about 15-20 minutes to answer 79 questions; therefore, KDQOL-SF ${ }^{\mathrm{TM}}$ is feasible in clinical research. The item scores were aggregated without weighting and transformed linearly to a 0-100 range, with higher scores indicating better statues.

\section{Statistical analysis}

Continuous variables were expressed as the mean and standard deviation, and categorical variables were presented as frequencies with percentages. All variables were tested for normal distribution using the KolmogorovSmirnov test. The effect of each KDQOL index gathered at 3 months after dialysis initiation on patient mortality was analyzed using the Kaplan-Meier survival graph and the Cox proportional hazard model. Each of the HRQOL indices is represented by a discontinuous number ranging from 0 to 100; these values are divided into tertiles, quartiles, and quintiles according to their characteristics. The hazard ratios (HR) and $95 \%$ confidence interval of each HRQOL index for overall mortality were calculated with the Cox analysis, comparing the significance of each index's effect on patient prognosis. Multivariable Cox analysis was performed after adjusting for age, sex, mCCI, and causes of primary renal disease. The IBM SPSS Sta- 
tistics software (version 22.0; IBM Corp., Armonk, NY, USA) was used for all analyses. A 2-tailed $P$ value $<0.05$ was considered significant.

\section{Results}

Patient characteristics according to physical and mental health status

The clinical and laboratory characteristics of the 568 adult dialysis patients included in the study according to physical and mental health status are shown in Table 1. Physical and mental health status was classified by upper and lower half of physical and mental health composite scores. All patients included in the analysis were hemodialysis patients. The mean age of the patients was $60.8 \pm 13.5$ years and $61.6 \%$ of the patients were male. The causes of ESKD were diabetes mellitus (56.9\%), hypertension (12.0\%), and glomerulonephritis (16.0\%). At the time of dialysis initiation, the mean eGFR was $6.5 \pm 3.4 \mathrm{~mL} /$

Table 1. Baseline characteristics according to physical and mental health status

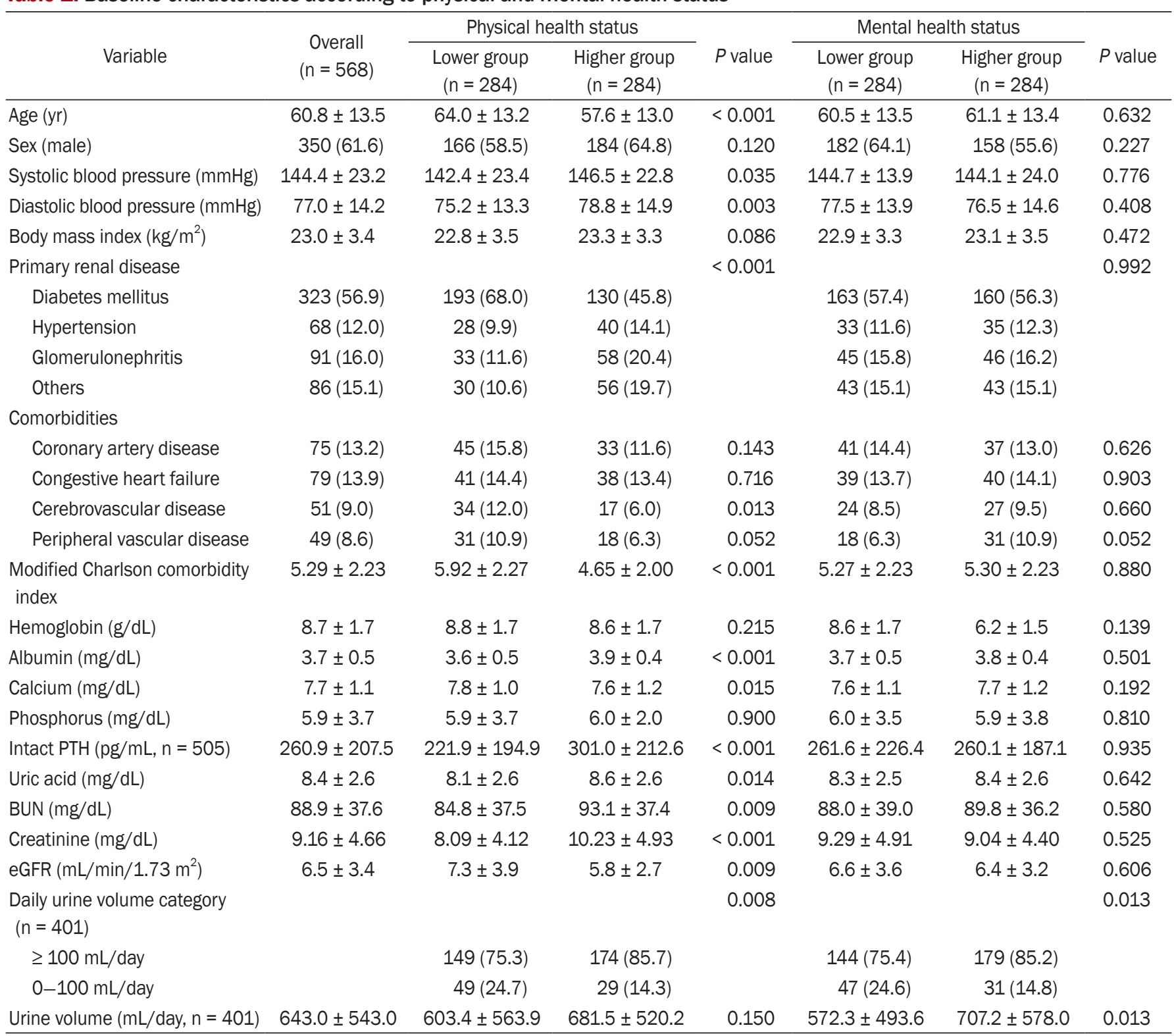

Data are presented as mean \pm standard deviation or number $(\%)$.

Physical and mental health status was classified by upper and lower half of physical and mental health composite scores.

BUN, blood urea nitrogen; GFR, glomerular filtration rate; PTH, parathyroid hormone. 

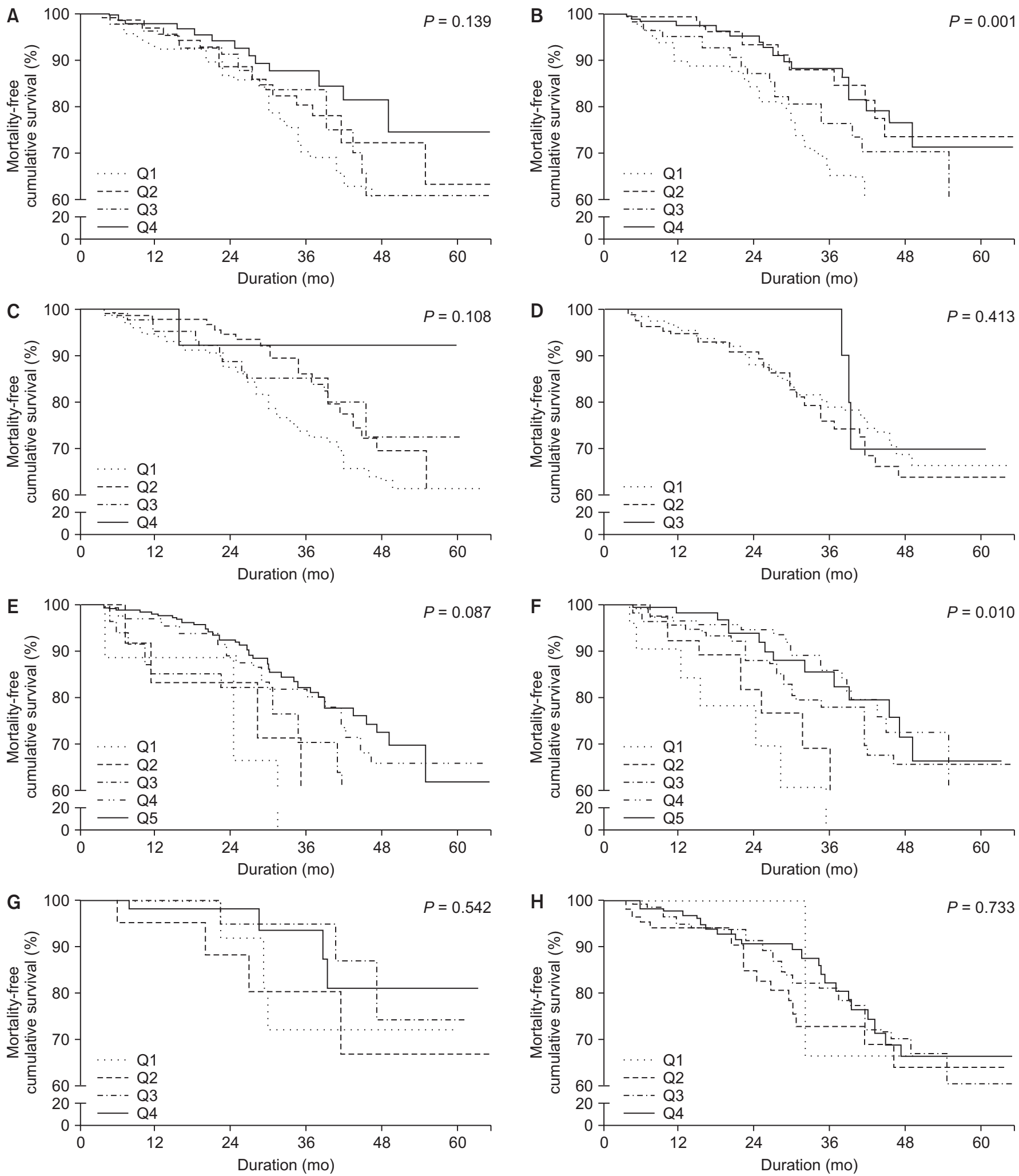

Figure 1. Patient survival according to the kidney disease elements, overall health, and patient satisfaction. (A) Symptoms/problem list. (B) Effects of kidney disease. (C) Burden of kidney disease. (D) Work status. (E) Cognitive function. (F) Quality of social interaction. (G) Sexual function. (H) Sleep. (I) Social support. (J) Dialysis staff encouragement. (K) Overall health. (L) Patient satisfaction. Among the various elements, lower scores of HRQOL including (B) effects of kidney disease $(P=0.001)$, (F) quality of social interaction $(P=0.010)$, (I) social support $(P=0.001)$, and $(J)$ dialysis staff encouragement $(P=0.028)$ were significantly associated with poor patient survival. Q1, Q2, Q3, Q4, and Q5 means 3- to 5-quantile group as appropriate. 

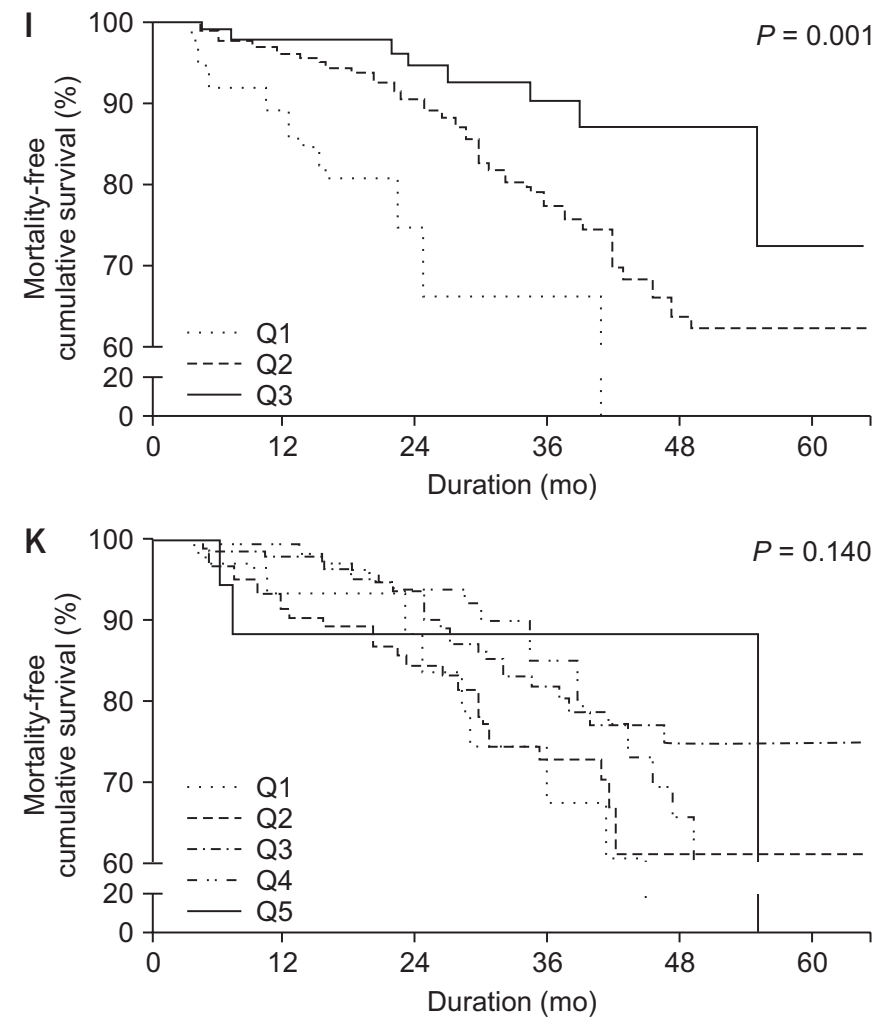

Figure 1. Continued.

$\min / 1.73 \mathrm{~m}^{2}$ and the mean hemoglobin level was $8.7 \pm$ $1.7 \mathrm{~g} / \mathrm{dL}$. Patients with poor physical health status tended to have older age, relatively lower blood pressure, higher prevalence of diabetes mellitus, higher comorbidities, lower serum albumin, higher eGFR, and higher prevalence of anuria (defined by urine output below $100 \mathrm{~mL} /$ day). In contrast, patients with poor mental health status showed no significance differences in almost all clinical parameters except urine volume. Low urine volume and anuria were significantly associated with poor mental health status $(P=0.013)$. Smoking status, serum glucose, hemoglobin Alc, total cholesterol, single-pool Kt/V, urea reduction rate, hemoglobin, serum iron, ferritin, and erythropoiesis-stimulating agent usage were not different according to physical and mental health status (Supplementary Table 1).

\section{HRQOL elements in kidney disease, overall health, and patient satisfaction}

Fig. 1 shows patient survival according to the HRQOL elements in kidney disease, overall health, and patient satisfaction domain and items. Among the various el-
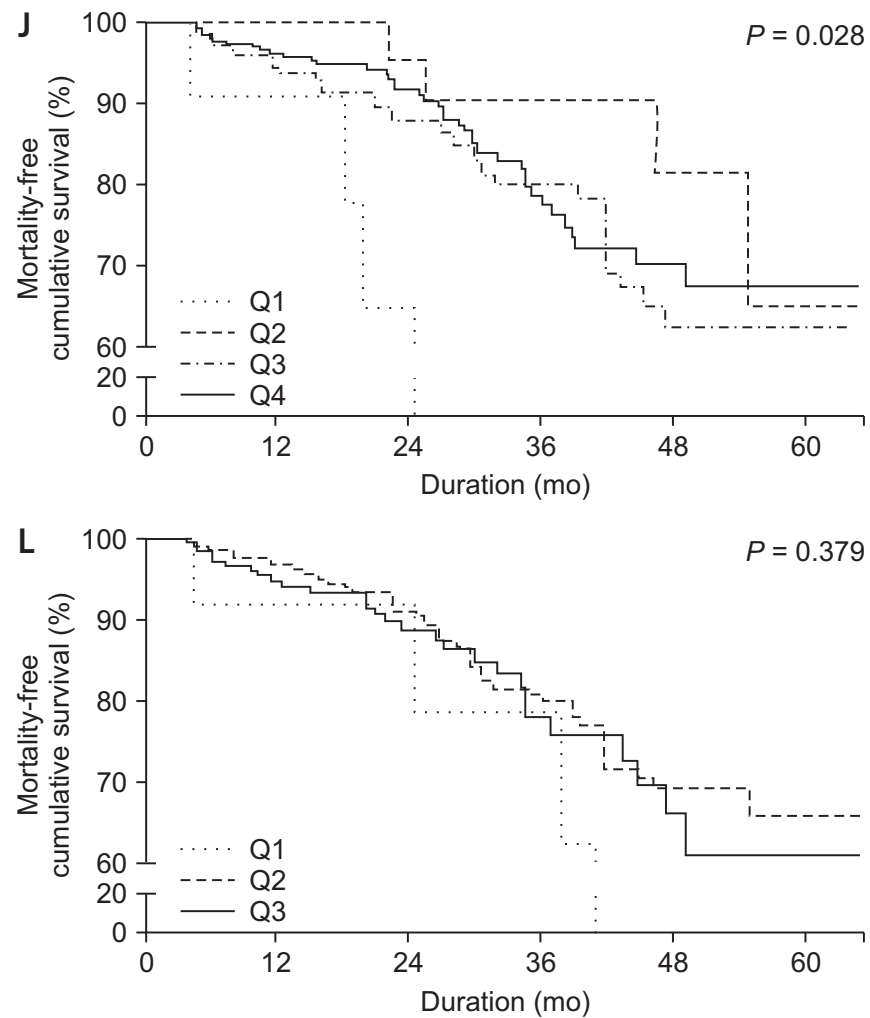

ements, lower scores of HRQOL, including effects of kidney disease $(P=0.001)$, quality of social interaction $(P=0.010)$, social support $(P=0.001)$, and dialysis staff encouragement $(P=0.028)$, were significantly associated with poor survival. Symptoms/problem list, burden of kidney disease, work status, cognitive function, sexual function, sleep, overall health, and patient satisfaction were not associated with mortality. Table 2 summarizes the results of univariable and multivariable proportional Cox hazard analysis of the HRQOL elements in the kidney disease domain. Effects of kidney disease, social support, and dialysis staff encouragement showed significant associations with mortality in both univariable and multivariable analysis. In particular, lower scores in the social support area were linearly associated with a higher risk of mortality.

\section{HRQOL elements in physical and mental health domain}

Fig. 2 shows patient survival according to the HRQOL elements in the physical health domain. Three of the four elements in the physical health domain, physical function, physical role limitation, and pain, were significantly 
Table 2. Risk of kidney disease-related elements of HRQOL on mortality

\begin{tabular}{|c|c|c|c|c|}
\hline & \multicolumn{2}{|c|}{ Univariable analysis } & \multicolumn{2}{|c|}{ Multivariable analysis } \\
\hline & $\operatorname{HR}(95 \% \mathrm{Cl})$ & $P$ value & $\mathrm{HR}(95 \% \mathrm{Cl})$ & $P$ value \\
\hline Effects of kidney disease & & 0.013 & & 0.014 \\
\hline Quartile 2 & $0.436(0.229-0.830)$ & 0.012 & $0.407(0.213-0.778)$ & 0.007 \\
\hline Quartile 3 & $0.718(0.410-1.257)$ & 0.246 & $0.812(0.455-1.449)$ & 0.482 \\
\hline Social support & & 0.002 & & $<0.001$ \\
\hline 0-33.3 (reference) & 1 & & 1 & \\
\hline $33.3-66.6$ & $0.416(0.206-0.839)$ & 0.014 & $0.282(0.135-0.588)$ & 0.001 \\
\hline \multirow[t]{2}{*}{$66.6-100$} & $0.178(0.068-0.463)$ & $<0.001$ & $0.117(0.042-0.324)$ & $<0.001$ \\
\hline & & $<0.001^{a}$ & & $<0.001^{a}$ \\
\hline $50-75$ & $0.354(0.138-0.909)$ & 0.031 & $0.252(0.093-0.681)$ & 0.007 \\
\hline \multirow[t]{2}{*}{$75-100$} & $0.298(0.117-0.756)$ & 0.011 & $0.198(0.074-0.527)$ & 0.001 \\
\hline & & $0.303^{\mathrm{a}}$ & & $0.059^{a}$ \\
\hline
\end{tabular}

$\mathrm{Cl}$, confidence interval; HR, hazard ratio; HRQOL, health-related quality of life.

${ }^{\mathrm{a} P}$ for trend.
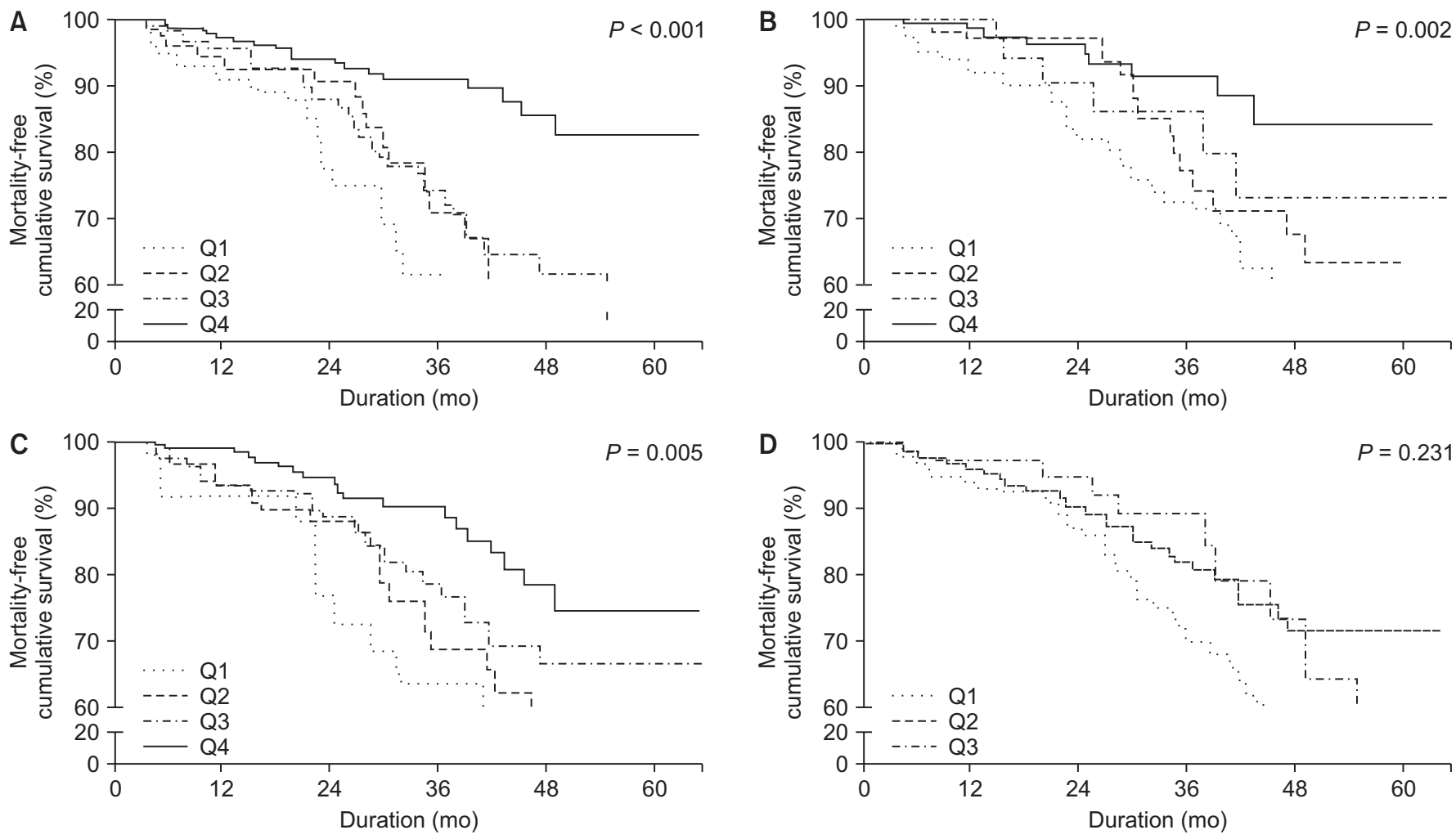

Figure 2. Patient survival according to the physical health domain elements. (A) Physical function. (B) Physical role limitation. (C) Pain. (D) General health. Among elements in the physical health domain, lower scores of HRQOL including $(A)$ physical function $(P<0.001)$, $(B)$ physical role limitation $(P=0.002)$, and $(C)$ pain $(P=0.005)$ were significantly associated with poor patient survival. Q1, Q2, Q3, Q4, and Q5 means 3- to 5- quantile group as appropriate. 
Table 3. Risk of physical health elements of HRQOL on mortality

\begin{tabular}{|c|c|c|c|c|}
\hline & \multicolumn{2}{|c|}{ Univariable analysis } & \multicolumn{2}{|c|}{ Multivariable analysis } \\
\hline & $\mathrm{HR}(95 \% \mathrm{Cl})$ & $P$ value & $\mathrm{HR}(95 \% \mathrm{Cl})$ & $P$ value \\
\hline Physical function & & $<0.001$ & & 0.029 \\
\hline 0-25 (reference) & 1 & & 1 & \\
\hline $25-50$ & $0.649(0.343-1.228)$ & 0.184 & $0.618(0.319-1.196)$ & 0.153 \\
\hline $50-75$ & $0.557(0.308-1.006)$ & 0.052 & $0.589(0.315-1.100)$ & 0.097 \\
\hline \multirow[t]{2}{*}{$75-100$} & $0.202(0.107-0.379)$ & $<0.001$ & $0.323(0.155-0.675)$ & 0.003 \\
\hline & & $<0.001^{a}$ & & $0.004^{a}$ \\
\hline Role limitation (physical) & & 0.002 & & 0.028 \\
\hline 0 (reference) & 1 & & 1 & \\
\hline $25-50$ & $0.571(0.326-1.003)$ & 0.051 & $0.566(0.314-1.020)$ & 0.058 \\
\hline $50-75$ & $0.503(0.216-1.173)$ & 0.112 & $0.676(0.284-1.607)$ & 0.375 \\
\hline \multirow[t]{2}{*}{$75-100$} & $0.285(0.140-0.579)$ & 0.001 & $0.369(0.179-0.761)$ & 0.007 \\
\hline & & $<0.001^{a}$ & & $0.005^{\mathrm{a}}$ \\
\hline Body pain & & 0.005 & & 0.017 \\
\hline 0-25 (reference) & 1 & & 1 & \\
\hline $25-50$ & $0.751(0.371-1.517)$ & 0.424 & $0.604(0.296-1.235)$ & 0.167 \\
\hline $50-75$ & $0.572(0.292-1.122)$ & 0.104 & $0.576(0.288-1.154)$ & 0.120 \\
\hline \multirow[t]{2}{*}{$75-100$} & $0.307(0.149-0.632)$ & 0.001 & $0.307(0.145-0.648)$ & 0.002 \\
\hline & & $<0.001^{\mathrm{a}}$ & & $0.002^{a}$ \\
\hline
\end{tabular}

$\mathrm{Cl}$, confidence interval; $\mathrm{HR}$, hazard ratio; HRQOL, health-related quality of life.

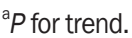

associated with mortality. Table 3 shows the results of univariable and multivariable proportional Cox hazard model analysis of the HRQOL elements in the physical health domain. Lower scores in physical function, physical role limitation, and body pain were associated with higher mortality and showed a linear trend of increased risk.

Fig. 3 depicts patient survival according to the HRQOL elements in the mental health domain. None of the 4 elements in the mental health domain were associated with patient mortality. In both univariable and multivariable Cox proportional hazard model analysis, all the elements in the mental health domain were not associated with higher mortality.

Fig. 4 illustrates patient survival according to the mental and physical composite scores. Lower levels in physical composite scores were significantly associated with mortality $(P<0.001)$. However, mental composite scores were not associated with increased risk for mortality $(P=$ 0.607). Table 4 shows the results of univariable and multivariable proportional Cox hazard model analysis of the physical and mental composite scores. Lower physical composite scores were associated with higher mortality and showed a linear trend of increased mortality.

\section{Effects of physical and mental health on survival according to age}

We also analyzed whether the effects of physical and mental composite scores on survival differed according to age groups. Table 5 shows the effects of HRQOL on survival in both the young or middle-aged group (age below 65 years old) and old-aged group (65 years old or above). Interestingly, in the old-aged group, both the physical and mental composite scores did not influence survival outcome. However, in the young or middle-aged group, physical components were significantly associated with survival outcomes.

\section{Discussion}

Dialysis patients generally have poor HRQOL compared with the general population or even other CKD patients [35]. HRQOL can be closely associated with patient prognosis, and the effect of each HRQOL component on prognosis can differ according to race. In this study, we inves- 

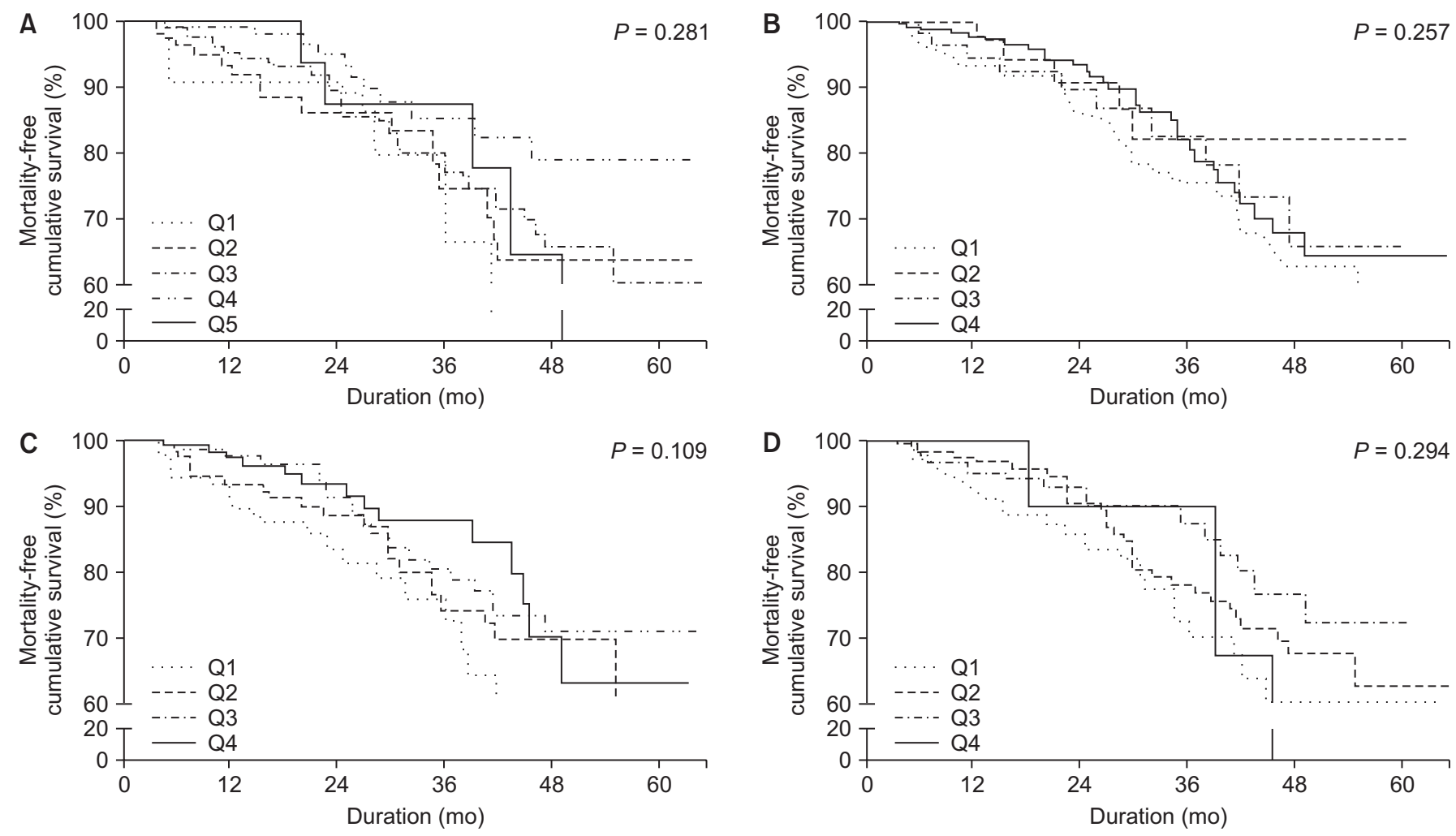

Figure 3. Patient survival according to the mental health domain elements. (A) Emotional well-being. (B) Emotional-role limitation. (C) Social function. (D) Energy/fatigue. All HRQOL elements in the mental health domain including $(A)$ emotional well-being $(P=0.281)$, $(B)$ emotional-role limitation $(P=0.257)$, $(C)$ social function $(P=0.109)$, and $(D)$ energy/fatigue $(P=0.294)$ were not significantly associated with patient survival. Q1, Q2, Q3, Q4, and Q5 means 3- to 5- quantile group as appropriate.
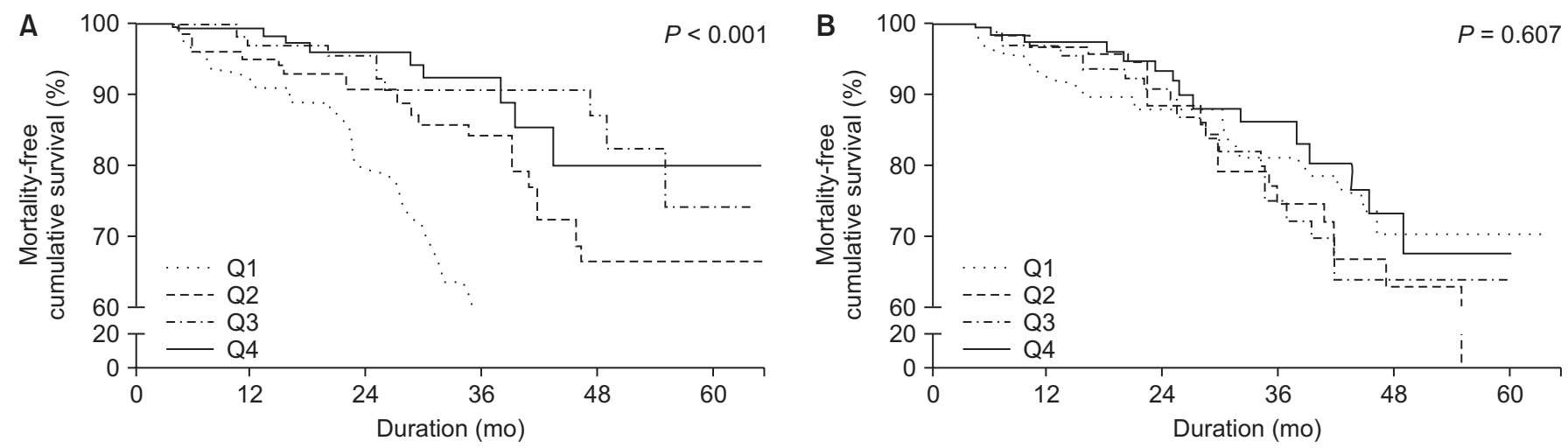

\begin{tabular}{|c|c|c|c|c|c|c|}
\hline $\begin{array}{r}\text { Numbers } \\
\text { at risk }\end{array}$ & 0 & 12 & 24 & 36 & 48 & 60 \\
\hline Q1 & 140 & 96 & 67 & 29 & 10 & 1 \\
\hline Q2 & 143 & 98 & 64 & 37 & 19 & 5 \\
\hline Q3 & 140 & 89 & 63 & 39 & 19 & 3 \\
\hline Q4 & 141 & 100 & 60 & 27 & 12 & 3 \\
\hline
\end{tabular}

$\begin{array}{rcr}\begin{array}{r}\text { Numbers } \\ \text { at risk }\end{array} & 0 & 12 \\ \text { Q1 } & 141 & 96 \\ \text { Q2 } & 139 & 95 \\ \text { Q3 } & 142 & 99 \\ \text { Q4 } & 142 & 93\end{array}$

$\begin{array}{llll}24 & 36 & 48 & 60 \\ 69 & 37 & 19 & 5 \\ 65 & 33 & 11 & 3 \\ 62 & 29 & 14 & 2 \\ 58 & 33 & 16 & 2\end{array}$

Figure 4. Patient survival according to the physical and mental composite scores. (A) Physical composite score. (B) Mental composite score. Lower physical composite scores $(P<0.001)$ were significantly associated with poor patient survival. Mental composite scores $(P=$ 0.607) were not associated with patient mortality. Q1, Q2, Q3, Q4, and Q5 means 3- to 5- quantile group as appropriate. 
Table 4. Risk of physical and mental composite score of HRQOL on mortality

\begin{tabular}{|c|c|c|c|c|}
\hline & \multicolumn{2}{|c|}{ Univariable analysis } & \multicolumn{2}{|c|}{ Multivariable analysis } \\
\hline & $\mathrm{HR}(95 \% \mathrm{Cl})$ & $P$ value & $\mathrm{HR}(95 \% \mathrm{Cl})$ & $P$ value \\
\hline Physical composite score & & $<0.001$ & & 0.003 \\
\hline Quartile 1 (reference) & 1 & & 1 & \\
\hline Quartile 2 & $0.454(0.268-0.768)$ & 0.003 & $0.535(0.312-0.916)$ & 0.023 \\
\hline Quartile 3 & $0.221(0.110-0.442)$ & $<0.001$ & $0.326(0.155-0.685)$ & 0.003 \\
\hline \multirow[t]{2}{*}{ Quartile 4} & $0.220(0.107-0.452)$ & $<0.001$ & $0.357(0.165-0.771)$ & 0.009 \\
\hline & & $<0.001^{\mathrm{a}}$ & & $0.001^{\mathrm{a}}$ \\
\hline Mental composite score & & 0.611 & & 0.660 \\
\hline Quartile 1 (reference) & 1 & & 1 & \\
\hline Quartile 2 & $1.195(0.670-2.133)$ & 0.546 & $0.963(0.532-1.741)$ & 0.900 \\
\hline Quartile 3 & $1.086(0.596-1.976)$ & 0.788 & $0.900(0.484-1.674)$ & 0.740 \\
\hline \multirow[t]{2}{*}{ Quartile 4} & $0.775(0.402-1.495)$ & 0.447 & $0.664(0.336-1.313)$ & 0.240 \\
\hline & & $0.457^{\mathrm{a}}$ & & $0.250^{\mathrm{a}}$ \\
\hline
\end{tabular}

$\mathrm{Cl}$, confidence interval; $\mathrm{HR}$, hazard ratio; $\mathrm{HRQOL}$, health-related quality of life.

${ }^{\mathrm{a} P}$ for trend.

Table 5. Adjusted risk of physical and mental composite score of HRQOL on mortality according to age

\begin{tabular}{|c|c|c|c|c|}
\hline & \multicolumn{2}{|c|}{ Age $<65$ years $(n=324)$} & \multicolumn{2}{|c|}{ Age $\geq 65$ years $(n=244)$} \\
\hline & $\mathrm{HR}(95 \% \mathrm{Cl})$ & $P$ value & $\mathrm{HR}(95 \% \mathrm{Cl})$ & $P$ value \\
\hline Physical composite score & & 0.005 & & 0.245 \\
\hline Quartile 1 (reference) & 1 & & 1 & \\
\hline Quartile 2 & $0.484(0.179-1.313)$ & 0.154 & $0.557(0.284-1.092)$ & 0.557 \\
\hline Quartile 3 & $0.121(0.025-0.598)$ & 0.010 & $0.497(0.209-1.181)$ & 0.113 \\
\hline \multirow[t]{2}{*}{ Quartile 4} & $0.136(0.036-0.508)$ & 0.003 & $0.692(0.261-1.831)$ & 0.458 \\
\hline & & $0.001^{a}$ & & $0.801^{a}$ \\
\hline Mental composite score & & 0.223 & & 0.777 \\
\hline Quartile 1 (reference) & 1 & & 1 & \\
\hline Quartile 2 & $1.078(0.417-2.784)$ & 0.877 & $0.845(0.377-1.890)$ & 0.681 \\
\hline Quartile 3 & $0.385(0.115-1.289)$ & 0.122 & $1.152(0.517-2.563)$ & 0.729 \\
\hline \multirow[t]{2}{*}{ Quartile 4} & $0.420(0.111-1.584)$ & 0.200 & $0.817(0.347-1.920)$ & 0.642 \\
\hline & & $0.071^{\mathrm{a}}$ & & $0.858^{a}$ \\
\hline
\end{tabular}

Multivariable Cox analysis was performed after adjusting for age, sex, modified Charlson comorbidity index, and causes of primary kidney disease.

$\mathrm{Cl}$, confidence interval; HR, hazard ratio; HRQOL, health-related quality of life.

${ }^{\text {a }} P$ for trend.

tigated the effect of HRQOL on the long-term survival of hemodialysis patients after the onset of dialysis using a prospective cohort of Asian incident dialysis patients. We enrolled a total of 568 hemodialysis patients who completed KDQOL-SF ${ }^{\mathrm{TM}} 1.3$ at 3 months after dialysis start. KDQOL-SF ${ }^{\mathrm{TM}} 1.3$ contains 3 main domains: the kidney disease-related domain (10 indices, patient satisfaction index, and overall health index), physical health domain (4 indices), and mental health domain (4 indices). The physical composite and mental composite scores can be obtained using items and indices belonging to the physi- cal health domain and mental health domain. In our study, the scores of 3 indices in the kidney disease domain (effect of kidney disease, social support, and dialysis staff encouragement) and the 3 indices in the physical health domain (physical function, physical role limitation, and body pain) showed significant associations with patient mortality, while a high physical composite score was associated with a decreased risk of patient mortality. The mental health indices and composite scores were not significantly associated with mortality.

A patient-reported outcome is a health outcome di- 
rectly reported by the patients without intervention or interpretation by a clinician or other medical staff. HRQOL is one of the most representative components of patient-reported outcomes. HRQOL can provide useful information on patients' subjective satisfaction or health status and can be a therapeutic target utilized in clinical practice or medical research. Periodically measured HRQOL using validated instruments might be more important than general expectations for patients on dialysis. These patients experience frequent physical and mental disabilities that can result in poor clinical outcomes [36]. Recently, investigators studied the role and potential application of HRQOLs in physical and mental health status using various questionnaire instruments on patients undergoing dialysis [37]. The KDQOL-SF 1.3 is a widely used and validated HRQOL instrument among dialysis patients [38]. The clinical factors associated with poor HRQOL, including old age, comorbidities, dialysis duration, and educational status, are relatively well studied among dialysis patients [39].

However, there is a lack of research on the effect of HRQOL on the prognosis of dialysis patients according to the HRQOL components including physical and mental health. McClellan et al. reported that the functional status and HRQOL measured by the Karnofsky Performance Scale and Spitzer Quality of Life Index were significantly associated with mortality in incident dialysis patients [40]. Peng et al [9] reported that poor physical dimension in HRQOL is a strong predictor of mortality among hemodialysis patients in Taiwan. In some studies, low scores on both physical and mental components were revealed as independent risk factors for higher mortality $[6,41]$. In contrast, other researchers reported that only mental health status was associated with higher mortality in dialysis patients $[4,42]$. In these studies, physical health scores were not associated with dialysis patient survival. Our results showing the prognostic impact of HRQOL only in the physical health domains on dialysis patient survival is in contrast with the previous studies that showed the association between mental health status and dialysis patient survival. Feroze et al [43] found that poor mental health scores were independently associated with higher mortality in all races and poor physical health scores were significantly associated with mortality only in the non-African American group. Therefore, the differential effect of physical and mental health compo- nents on prognosis might be derived from the different characteristics of the study population or ethnicities.

Recently, Kalantar et al [44] reported the impact of ethnicity on the association between HRQOL and mortality among dialysis patients in the US. When patients were stratified by ethnicity, physical composite scores were significantly associated with poor overall survival in all ethnicity groups, and Hispanic and patients of other ethnicities showed a relatively higher mortality risk compared with African American dialysis patients. However, mental composite scores were not associated with increased mortality according to each ethnicity group. These results are similar to our research. In general, African American and Hispanic dialysis patients show better survival compared with Caucasian dialysis patients [45]. The researchers found that the survival advantage of African American and Hispanic dialysis patients disappeared after adjusting for nutritional and inflammatory conditions and that the risk of death among African American dialysis patients was even greater [46]. Good nutritional status is known to correlate well with high HRQOL scores $[47,48]$. The superiority of nutritional and inflammatory status among African American and Hispanic dialysis patients might be a possible explanation for the nonassociation between physical health and survival in these populations. Other causes of racial differences in dialysis patient survival according to HRQOL were suggested, including differences in degrees of familial and social support, medical insurance, accessibility to transplantation, policy and treatment guidelines for dialysis therapy, dialysis access type, and cardiovascular risk and anthropometric profile [49]. Further studies are needed to determine whether there is a difference in the prevalence of depression, mental health status, and their survival effects in dialysis patients according to different ethnicities.

Our study also showed that the impact of physical health status on survival was still significant in the young and middle-aged groups. However, in the subgroup analysis of the old age group, the significance of physical health status on survival was not sustained. Aged patients on dialysis are more often frail and have more comorbidities and greater functional impairment than younger dialysis patients [50]. Since dialysis is an intensive treatment, its suitability in older patients has been questioned [51]. Non-dialytic conservative care has been proposed as an alternative to dialysis for selected older 
patients with ESKD. A recent systematic review showed that conservative care has the potential to achieve similar HRQOL in select aged dialysis patients [52]. The results of our study support the importance of monitoring physical health status and the achievement of good functional activities during the dialysis treatment process, especially in the young or middle-aged group.

Despite the aforementioned advantages, there are several limitations in this study. First, potential prognostic factors that can have differential effects on survival outcomes in dialysis patients according to ethnicity, including inflammatory markers, directly measured muscle mass, or nutritional status indices, were not considered in this study. Instead, our study analyzed the different characteristics according to the physical and mental health status. Although several clinical factors including old age, comorbidities, low serum albumin levels, and anuria were associated with poor physical health status, mental health status was only associated with decreased urine volume or anuria. Second, in this study, only the HRQOLs at 3 months after dialysis start were analyzed. Recently, Moore et al [53] reported that HRQOL improves during the 3 month transition period after dialysis start, especially in physical domain. Whether HRQOL at predialysis or 3 months post-dialysis is more important for survival and whether HRQOL improvement can influence patient mortality are important questions. Further studies according to the prognostic values of HRQOLs at different time points and subsequent HRQOL improvement or deterioration are warranted. Lastly, this study enrolled only hemodialysis patients, and the results cannot easily be generalized to peritoneal dialysis patients. Several previous studies have not found any differences in HRQOL in non-Asian dialysis patients according the hemodialysis and peritoneal dialysis treatment modality [54,55]. In an investigation in Singapore, patients with peritoneal dialysis showed a higher prevalence of depression and poor physical health status [56]. In contrast, some reports indicated that peritoneal dialysis patients showed better HRQOL especially in other Asian populations. Zhang et al [57] found that peritoneal dialysis patients reported higher scores of HRQOL, especially in mental health domains, and comparable physical function status in a Chinese dialysis population. Hsu et al [58] reported that peritoneal dialysis patients had better HRQOL even after adjusting the dialysis duration in a multi-center prospective cross- sectional study in Taiwan. Recently, Chuasuwan et al [59] performed a meta-analysis according to dialysis modality and HRQOL and reported that peritoneal dialysis patients had better HRQOL. Whether the effect of physical and mental health domains of HRQOL on survival might differ according to dialysis modality, especially in Asian dialysis patients, should be investigated.

In conclusion, poor physical health at 3 months after dialysis initiation correlates significantly with overall mortality in patients on hemodialysis. Among 12 indices in the kidney disease domain, 3 indices showed significant associations with patient mortality, and 3 indices in the physical health domain showed significant associations with patient mortality. In contrast, all 4 indices in the mental health domain did not show a significant association with mortality, and the mental composite score also did not show a significant association with mortality. Our findings underscore the importance of HRQOL on hemodialysis patients' overall prognosis and suggest that improving physical health status through interventions could increase patient survival.

\section{Conflicts of interest}

All authors have no conflicts of interest to declare.

\section{Funding}

This research was supported by a grant of the Korea Health Technology R\&D Project through the Korea Health Industry Development Institute (KHIDI), funded by the Ministry of Health \& Welfare, Republic of Korea (grant number: HC15C1129).

\section{Acknowledgments}

A part of this study was presented in the 38th annual meeting of the Korean Society of Nephrology (Oral Presentation; May 17, 2018). We gratefully acknowledge the dedication of the clinical research coordinators and medical staff members of the participating centers. The authors would like to thank the generosity of the study participants. 


\section{Authors' contributions}

Yong-Lim Kim, Yon Su Kim, and Jung Pyo Lee developed the research idea and designed this study. Yong Chul Kim, Soie Kwon, Lilin Li, Do Hyoung Kim, Jung Nam An, Jang-Hee Cho, Dong Ki Kim, Yun Kyu Oh, and Chun Soo Lim acquired the data. Jeonghwan Lee, Sohee Oh, Jung Nam An, and Jung Pyo Lee analyzed the data. Jeonghwan Lee and Jung Pyo Lee wrote the manuscript. JangHee Cho and Chun Soo Lim supervised the manuscript.

\section{References}

[1] Demura S, Sato S. Relationships between depression, lifestyle and quality of life in the community dwelling elderly: a comparison between gender and age groups. J Physiol Anthropol Appl Human Sci 2003;22:159-166.

[2] Chen SS, Al Mawed S, Unruh M. Health-related quality of life in end-stage renal disease patients: how often should we ask and what do we do with the answer? Blood Purif 2016;41:218-224.

[3] Kalantar-Zadeh K, Unruh M. Health related quality of life in patients with chronic kidney disease. Int Urol Nephrol 2005;37:367-378.

[4] Kalantar-Zadeh K, Kopple JD, Block G, Humphreys MH. Association among SF36 quality of life measures and nutrition, hospitalization, and mortality in hemodialysis. J Am Soc Nephrol 2001;12:2797-2806.

[5] Fukuhara S, Lopes AA, Bragg-Gresham JL, et al. Healthrelated quality of life among dialysis patients on three continents: the Dialysis Outcomes and Practice Patterns Study. Kidney Int 2003;64:1903-1910.

[6] Lowrie EG, Curtin RB, LePain N, Schatell D. Medical outcomes study short form-36: a consistent and powerful predictor of morbidity and mortality in dialysis patients. Am J Kidney Dis 2003;41:1286-1292.

[7] Grubbs V, Tuot DS, Powe NR, O'Donoghue D, Chesla CA. System-level barriers and facilitators for foregoing or withdrawing dialysis: a qualitative study of nephrologists in the United States and England. Am J Kidney Dis 2017;70:602610.

[8] Valdés C, García-Mendoza M, Rebollo P, Ortega T, Ortega F. Mental health at the third month of haemodialysis as a predictor of short-term survival. Nephrol Dial Transplant 2006;21:3223-3230.

[9] Peng YS, Chiang CK, Hung KY, et al. Are both psychological and physical dimensions in health-related quality of life associated with mortality in hemodialysis patients: a 7-year Taiwan cohort study. Blood Purif 2010;30:98-105.

[10] Dukkipati R, Kalantar-Zadeh K, Kopple JD. Is there a role for intradialytic parenteral nutrition? A review of the evidence. Am J Kidney Dis 2010;55:352-364.

[11] Cohen SD, Norris L, Acquaviva K, Peterson RA, Kimmel PL. Screening, diagnosis, and treatment of depression in patients with end-stage renal disease. Clin J Am Soc Nephrol 2007;2:1332-1342.

[12] Hedayati SS, Bosworth HB, Briley LP, et al. Death or hospitalization of patients on chronic hemodialysis is associated with a physician-based diagnosis of depression. Kidney Int 2008;74:930-936.

[13] Sohn BK, Oh YK, Choi JS, et al. Effectiveness of group cognitive behavioral therapy with mindfulness in end-stage renal disease hemodialysis patients. Kidney Res Clin Pract 2018;37:77-84

[14] Huang M, Lv A, Wang J, et al. Exercise training and outcomes in hemodialysis patients: systematic review and meta-analysis. Am J Nephrol 2019;50:240-254.

[15] Painter PL, Agarwal A, Drummond M. Physical function and physical activity in peritoneal dialysis patients. Perit Dial Int 2017;37:598-604.

[16] Kopple JD, Kim JC, Shapiro BB, et al. Factors affecting daily physical activity and physical performance in maintenance dialysis patients. J Ren Nutr 2015;25:217-222.

[17] Findlay MD, Mark PB. Reduced and declining physical function in prevalent dialysis patients-identifying the vulnerable. Age Ageing 2017;46:541-543.

[18] Unruh M, Miskulin D, Yan G, et al. Racial differences in health-related quality of life among hemodialysis patients. Kidney Int 2004;65:1482-1491.

[19] Hicks LS, Cleary PD, Epstein AM, Ayanian JZ. Differences in health-related quality of life and treatment preferences among black and white patients with end-stage renal disease. Qual Life Res 2004;13:1129-1137.

[20] Kalantar-Zadeh K, Golan E, Shohat T, Streja E, Norris KC, Kopple JD. Survival disparities within American and Israeli dialysis populations: learning from similarities and distinctions across race and ethnicity. Semin Dial 2010;23:586594.

[21] Agodoa L. Racial disparities in kidney health: the puzzle to solve. Am J Kidney Dis 2002;40:1337-1339.

[22] Jung HY, Choi H, Choi JY, et al. Dialysis modality-related disparities in sudden cardiac death: hemodialysis versus 
peritoneal dialysis. Kidney Res Clin Pract 2019;38:490-498.

[23] Zazzeroni L, Pasquinelli G, Nanni E, Cremonini V, Rubbi I. Comparison of quality of life in patients undergoing hemodialysis and peritoneal dialysis: a systematic review and meta-analysis. Kidney Blood Press Res 2017;42:717-727.

[24] Farag YM, Keithi-Reddy SR, Mittal BV, et al. Anemia, inflammation and health-related quality of life in chronic kidney disease patients. Clin Nephrol 2011;75:524-533.

[25] Klersy C, Callegari A, Giorgi I, Sepe V, Efficace E, Politi P. Italian translation, cultural adaptation and validation of KDQOL-SF, version 1.3, in patients with severe renal failure. J Nephrol 2007;20:43-51.

[26] Lee J, Lee JP, Park JI, et al. Early nephrology referral reduces the economic costs among patients who start renal replacement therapy: a prospective cohort study in Korea. PLoS One 2014;9:e99460.

[27] Kim DH, Kim M, Kim H, et al. Early referral to a nephrologist improved patient survival: prospective cohort study for end-stage renal disease in Korea. PLoS One 2013;8:e55323.

[28] Stevens LA, Schmid CH, Greene T, et al. Comparative performance of the CKD Epidemiology Collaboration (CKDEPI) and the Modification of Diet in Renal Disease (MDRD) Study equations for estimating GFR levels above $60 \mathrm{~mL} /$ min/1.73 m2. Am J Kidney Dis 2010;56:486-495.

[29] Levey AS, Stevens LA. Estimating GFR using the CKD Epidemiology Collaboration (CKD-EPI) creatinine equation: more accurate GFR estimates, lower CKD prevalence estimates, and better risk predictions. Am J Kidney Dis 2010;55: 622-627.

[30] Chae JW, Song CS, Kim H, Lee KB, Seo BS, Kim DI. Prediction of mortality in patients undergoing maintenance hemodialysis by Charlson Comorbidity Index using ICD-10 database. Nephron Clin Pract 2011;117:c379-c384.

[31] Charlson ME, Pompei P, Ales KL, MacKenzie CR. A new method of classifying prognostic comorbidity in longitudinal studies: development and validation. J Chronic Dis 1987;40:373-383.

[32] Park HJ, Kim S, Yong JS, et al. Reliability and validity of the Korean version of Kidney Disease Quality of Life instrument (KDQOL-SF). Tohoku J Exp Med 2007;211:321-329.

[33] Korevaar JC, Merkus MP, Jansen MA, Dekker FW, Boeschoten EW, Krediet RT. Validation of the KDQOL-SF: a dialysis-targeted health measure. Qual Life Res 2002;11:437447.

[34] Abd ElHafeez S, Sallam SA, Gad ZM, et al. Cultural adaptation and validation of the "Kidney Disease and Quality of
Life--Short Form (KDQOL-SF ${ }^{\mathrm{TM}}$ ) version 1.3" questionnaire in Egypt. BMC Nephrol 2012;13:170.

[35] Mujais SK, Story K, Brouillette J, et al. Health-related quality of life in CKD Patients: correlates and evolution over time. Clin J Am Soc Nephrol 2009;4:1293-1301.

[36] Fischer MJ, Porter AC, Lash JP. Treatment of depression and poor mental health among patients receiving maintenance dialysis: are there options other than a pill or a couch? Am J Kidney Dis 2013;61:694-697.

[37] Nayana SA, Balasubramanian T, Nathaliya PM, Nimsha Hussain P, Mohammed Salim KT, Muhammed Lubab P. A cross sectional study on assessment of health related quality of life among end stage renal disease patients undergoing hemodialysis. Clin Epidemiol Glob Health 2017;5:148153.

[38] Joshi VD, Mooppil N, Lim JF. Validation of the kidney disease quality of life-short form: a cross-sectional study of a dialysis-targeted health measure in Singapore. BMC Nephrol 2010;11:36.

[39] Mousa I, Ataba R, Al-ali K, Alkaiyat A, Zyoud SeH. Dialysisrelated factors affecting self-efficacy and quality of life in patients on haemodialysis: a cross-sectional study from Palestine. Ren Replace Ther 2018;4:21.

[40] McClellan WM, Anson C, Birkeli K, Tuttle E. Functional status and quality of life: predictors of early mortality among patients entering treatment for end stage renal disease. $J$ Clin Epidemiol 1991;44:83-89.

[41] Grincenkov FR, Fernandes N, Pereira Bdos S, et al. Impact of baseline health-related quality of life scores on survival of incident patients on peritoneal dialysis: a cohort study. Nephron 2015;129:97-103.

[42] López Revuelta K, García López FJ, de Alvaro Moreno F, Alonso J. Perceived mental health at the start of dialysis as a predictor of morbidity and mortality in patients with endstage renal disease (CALVIDIA Study). Nephrol Dial Transplant 2004;19:2347-2353.

[43] Feroze U, Noori N, Kovesdy CP, et al. Quality-of-life and mortality in hemodialysis patients: roles of race and nutritional status. Clin J Am Soc Nephrol 2011;6:1100-1111.

[44] Kalantar SS, You AS, Norris KC, et al. The impact of race and ethnicity upon health-related quality of life and mortality in dialysis patients. Kidney Med 2019;1:253-262.

[45] Kalantar-Zadeh K, Kovesdy CP, Norris KC. Racial survival paradox of dialysis patients: robust and resilient. Am J Kidney Dis 2012;60:182-185.

[46] Streja E, Kovesdy CP, Molnar MZ, et al. Role of nutritional 
status and inflammation in higher survival of African American and Hispanic hemodialysis patients. Am J Kidney Dis 2011;57:883-893.

[47] Rambod M, Bross R, Zitterkoph J, et al. Association of Malnutrition-Inflammation Score with quality of life and mortality in hemodialysis patients: a 5-year prospective cohort study. Am J Kidney Dis 2009;53:298-309.

[48] Kalantar-Zadeh K, Streja E, Kovesdy CP, et al. The obesity paradox and mortality associated with surrogates of body size and muscle mass in patients receiving hemodialysis. Mayo Clin Proc 2010;85:991-1001.

[49] Yan G, Norris KC, Yu AJ, et al. The relationship of age, race, and ethnicity with survival in dialysis patients. Clin J Am Soc Nephrol 2013;8:953-961.

[50] Berger JR, Hedayati SS. Renal replacement therapy in the elderly population. Clin J Am Soc Nephrol 2012;7:10391046.

[51] Farrington K, Covic A, Nistor I, et al. Clinical Practice Guideline on management of older patients with chronic kidney disease stage $3 \mathrm{~b}$ or higher $(\mathrm{eGFR}<45 \mathrm{~mL} / \mathrm{min} / 1.73$ $\mathrm{m} 2$ ): a summary document from the European Renal Best Practice Group. Nephrol Dial Transplant 2017;32:9-16.

[52] Verberne WR, van den Wittenboer ID, Voorend CGN, et al. Health-related quality of life and symptoms of conservative care versus dialysis in patients with end-stage kidney disease: a systematic review. Nephrol Dial Transplant 2020 Jun 14 [Epub]. Doi: 10.1093/ndt/gfaa078.

[53] Moore C, Carter LA, Mitra S, Skevington S, Wearden A.
Quality of life improved for patients after starting dialysis but is impaired, initially, for their partners: a multi-centre, longitudinal study. BMC Nephrol 2020;21:185.

[54] Wasserfallen JB, Moinat M, Halabi G, et al. Satisfaction of patients on chronic haemodialysis and peritoneal dialysis. Swiss Med Wkly 2006;136:210-217.

[55] Wu AW, Fink NE, Marsh-Manzi JV, et al. Changes in quality of life during hemodialysis and peritoneal dialysis treatment: generic and disease specific measures. J Am Soc Nephrol 2004;15:743-753.

[56] Griva K, Kang AW, Yu ZL, et al. Quality of life and emotional distress between patients on peritoneal dialysis versus community-based hemodialysis. Qual Life Res 2014;23:5766.

[57] Zhang AH, Cheng LT, Zhu N, Sun LH, Wang T. Comparison of quality of life and causes of hospitalization between hemodialysis and peritoneal dialysis patients in China. Health Qual Life Outcomes 2007;5:49.

[58] Hsu CC, Huang CC, Chang YC, Chen JS, Tsai WC, Wang KY. A comparison of quality of life between patients treated with different dialysis modalities in Taiwan. PLoS One 2020; 15:e0227297.

[59] Chuasuwan A, Pooripussarakul S, Thakkinstian A, Ingsathit A, Pattanaprateep O. Comparisons of quality of life between patients underwent peritoneal dialysis and hemodialysis: a systematic review and meta-analysis. Health Qual Life Outcomes 2020;18:191. 